\title{
Harnessing local and systemic immunity for vaccines against tuberculosis
}

\author{
PCL Beverley ${ }^{1}$, S Sridhar ${ }^{2}$, A Lalvani ${ }^{2}$ and EZ Tchilian ${ }^{1}$
}

The lung is the portal of entry for Mycobacterium tuberculosis (Mtb) and animal experimental evidence indicates that local immune defense mechanisms are crucial for protective immunity. Immunization via the lower respiratory tract efficiently induces a dividing, activated, antigen-dependent, lung-resident, memory T-cell population, which is partly recoverable by bronchoalveolar lavage. These cells can inhibit the growth of $M t b$ in the lungs immediately after infection. Delivery of appropriate signals to the lung innate immune system is critical for induction of effective local immunity. In contrast after parenteral immunization, antigen-specific cells may be found in lung tissue but few are recoverable by lavage and inhibition of mycobacterial growth is delayed. Harnessing both local and systemic immunity can provide highly effective protection in animal models and the evidence suggests that taken in aggregate, multiple animal models may predict the success of novel vaccine strategies in humans.

\section{INTRODUCTION}

Tuberculosis (TB) remains an important cause of morbidity and mortality worldwide, with 8.7 million new infections and 1.4 million deaths annually and in view of the spread of drugresistant organisms, new measures to contain the disease are urgently needed. In this light, the lack of efficacy of the recombinant vaccinia virus booster vaccine, MVA85A, in a recent phase IIB trial in infants is a significant setback to TB vaccine research, ${ }^{1,2}$ although much has been learnt about the conduct of a proof-of-concept efficacy trial in a high TB burden setting. ${ }^{3}$ Furthermore, although there are currently many other vaccine candidates in clinical trials, most of which induce similar immune responses to MVA85A, because of the lack of a protective immune correlate there is no certainty that any of these parenteral vaccines will be effective. ${ }^{4}$ Therefore, continuing development of new strategies and a better understanding of the nature of protective immunity remain important goals for TB research. Here we review the nature of protective immunity to Mycobacterium tuberculosis (Mtb) and discuss a potentially novel strategy for improving immunization against $\mathrm{TB}$, the harnessing of local lung immunity as well as systemic immunity.

DELAYED INITIATION OF THE IMMUNE RESPONSE TO MTB Few parenteral subunit vaccines when used alone show greater protective efficacy than Bacille Calmette Guerin (BCG) in animal models and even fewer provide a convincing increase in protection over BCG when used in prime/boost regimes. ${ }^{4}$ Furthermore, there is no correlation between the magnitude of peripheral blood mononuclear cell immune responses and protection. Thus, for example, a single parenteral boost with MVA85A after BCG priming is highly immunogenic in animals $^{5-9}$ and humans. ${ }^{2,10}$ However, although in two of these studies, in cows ${ }^{7}$ and primates, ${ }^{8}$ there is a trend toward improved protection in MVA85A boosted animals compared with BCG alone, the difference does not reach statistical significance, whereas in mice ${ }^{5}$ and guinea pigs ${ }^{6}$ there is no trend toward improved protection and in a second primate experiment, BCG primed MVA85A boosted monkeys did not differ from naive, unimmunized animals. ${ }^{9}$

Parenteral vaccine regimes that do induce improved protection over BCG alone frequently utilize multiple immunizations, which induces entry of cells into non-lymphoid tissues. ${ }^{11,12}$ This suggests that protection induced by effective parenteral regimes may depend not only on the quality of the response $\mathrm{e}^{13-16}$ but also the migratory properties of the antigen-specific cells and their role at the site of entry of $M t b$ in the lungs.

On the basis of the development of skin test reactivity, a measurable adaptive immune response to $M t b$ takes 6 weeks after exposure or infection to emerge (reviewed by Ernst ${ }^{17}$ ). In

${ }^{1}$ Nuffield Department of Medicine, University of Oxford, Oxford, UK and ${ }^{2}$ TB Research Unit, National Heart and Lung Institute, Imperial College, London, UK. Correspondence: PCL Beverley (peter.beverley@ndm.ox.ac.uk)

Received 29 August 2013; revised 9 October 2013; accepted 14 October 2013; published online 20 November 2013. doi:10.1038/mi.2013.99 


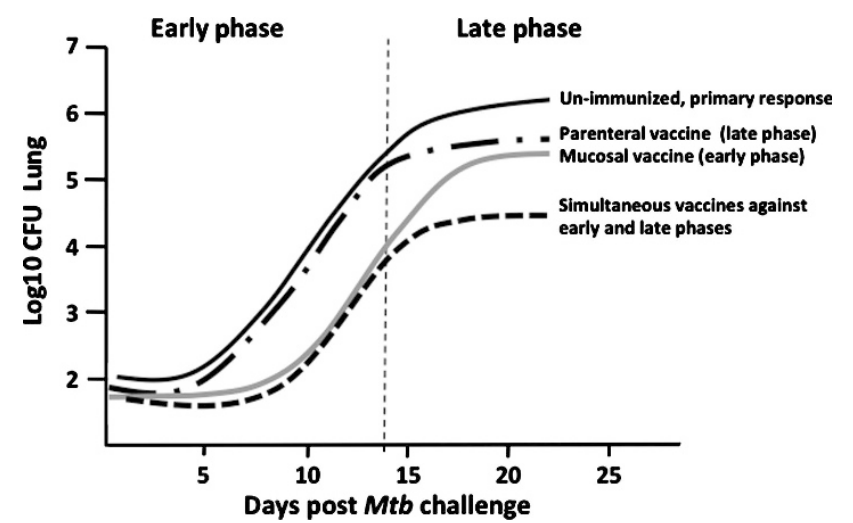

Figure 1 Schematic of Mycobacterium tuberculosis (Mtb) growth after challenge in mice. Un-immunized mice show an initial lag followed by logarithmic growth in colony forming units (CFU) and a plateau when a primary response to Mtb occurs. Mice immunized parenterally show identical kinetics of $M t b$ growth until $\sim 14$ days when further growth is inhibited (late phase immunity), while mucosal immunization inhibits Mtb growth early after challenge (early phase immunity), followed by logarithmic growth and eventual containment. Simultaneous immunization by both routes leads to inhibition of $M t b$ growth in both early and late phases of infection. The schematic is based on previously published data. ${ }^{22,39}$

mice too, the immune response to mycobacteria introduced into the lungs is delayed. This may be because $M t b$ deploys immune evasion strategies or because the initial exposure is to a low dose of organisms. ${ }^{18}$ Whatever the reason, it has been shown in mice that it is 8-10 days after infection before activated $\mathrm{T}$ cells can be detected in the draining mediastinal nodes and 12-14 days before they can be detected in the lungs. ${ }^{19-21}$ Until this time, after an initial lag phase, $M t b$ grows logarithmically, but the appearance of $\mathrm{T}$ cells in the lungs correlates with inhibition of growth, indicating their importance in controlling infection. Remarkably, in mice immunized parenterally with protective vaccines, including BCG, the kinetics of $M t b$ growth are identical up to $\sim 12$ days, although the mycobacterial load then stabilizes at a lower level than in naive animals ${ }^{1920,22,23}$ (Figure 1). Interestingly, in mice immunized parenterally with BCG, experiments using fingolimod, which inhibits egress of cells from lymph nodes, suggest that containment of challenge mycobacteria may not be due to an influx of cells from the periphery but rather to expansion of $\mathrm{T}$ cells already present in lung tissue. ${ }^{24}$

Irrespective of the origin of cells mediating immunity after parenteral immunization, it appears that in the mouse, systemic immunity, although it may confer a degree of containment, does not prevent establishment of infection and allows $M t b$ to grow unchecked for $>10$ days. There is therefore a period after lung infection, which is affected minimally or inefficiently by parenteral immunization (Figure 1). This period presents a challenge, or alternatively a window of opportunity, for vaccine strategies. Control of the early (first 10 days) phase of $M t b$ growth is likely to require local lung immune responses and therefore a better understanding of mucosal immunity will be needed in order to design effective mucosal vaccines.

\section{LOCAL IMMUNITY TO MTB}

\section{Tissue-resident $T$ cells}

In recent years, it has become clear that a large proportion of immune cells reside outside lymphoid organs and that tissueresident populations of lymphocytes are present in most organs. ${ }^{25}$ In mice, it has been demonstrated for acute infections, such as influenza, Sendai virus or respiratory syncytial virus, that lung-resident $\mathrm{T}$ memory cells $\left(\mathrm{T}_{\mathrm{RM}}\right)$ with specialized phenotypic and functional properties, ${ }^{26}$ have an important role in protection against challenge, whereas T-cell memory detected in blood or lymphoid organs does not correlate well with protection. ${ }^{27,28}$ Direct proof that $\mathrm{T}_{\mathrm{RM}}$ are crucial for protective immunity in humans is more difficult to obtain. However, human lung-resident $\mathrm{T}$ cells differ in many respects from those present in peripheral blood and are enriched for memory cells able to respond to common respiratory pathogens such as influenza virus. ${ }^{29}$ Furthermore, bronchoalveolar lavage (BAL) samples of purified protein derivative (PPD) skin test positive humans, with presumed latent $\mathrm{TB}$ infection, also contain PPD-reactive memory T cells. ${ }^{30}$

\section{Upper and lower respiratory tract immunity}

In the mouse, $\mathrm{T}$ cells responsible for immunity against respiratory virus infections are most effectively induced by intranasal immunization, with an inoculum volume that induces both upper and lower respiratory tract (URT and LRT) cellular immunity. ${ }^{27}$ However, URT and LRT immunity are at least partially separable in mice, as deliberate immunization of the URT only, although it induces a nasal-associated lymphoid tissue response, does not induce LRT $\mathrm{T}_{\mathrm{RM}}$. Furthermore, only LRT immunization generating LRT $T_{R M}$, is protective against $M t b$ challenge. ${ }^{31}$ Similarly, in macaques immunization with a $4 \mu \mathrm{m}$ particle size aerosol, which delivers antigen to the LRT, was shown to induce much greater lung immune responses than intranasal immunization, suggesting that in this species, as in mice, URT and LRT immune responses are distinct. ${ }^{32}$ It is not known how closely humans approximate to primates in this regard, as a human common respiratory tract mucosal system has been proposed, ${ }^{33,34}$ but its existence will only be established by systematic comparison of immune responses in the URT and LRT after deliberate immunization by one or the other route.

\section{Lung immunity to Mtb, the mouse model}

LRT immunization against $M t b$ has been intensively investigated in mice. Thus, for example, LRT immunization with recombinant adenoviruses expressing $M t b$ antigen $85 \mathrm{~A}$ (Ad85A) can generate protection equivalent to parenteral BCG and can also boost protection after parenteral BCG priming. ${ }^{35-37}$ Boosting with MVA85A intranasally, albeit after intranasal priming with BCG, also significantly improves protection compared with BCG alone. ${ }^{38}$ Kinetic studies of $M t b$ growth in the lungs of mice immunized with Ad85A provide an explanation for the efficacy of this route of immunization. Thus, $M t b$ growth is inhibited immediately after challenge in immunized mice and although after $\sim 1$ week $M t b$ escapes and grows logarithmically, the mycobacterial load stabilizes at a 
lower level than in naive mice ${ }^{39}$ (Figure 1). Similarly, after respiratory tract or parenteral immunization with BCG or recombinant $M t b$ antigens, early inhibition of growth of $M t b$ was demonstrated only in LRT immunized mice. ${ }^{22}$

Investigation of the location of antigen-specific cells after LRT immunization has shown that antigen-specific $\mathrm{T}$ cells are found in both lung interstitial tissue and at a high frequency in BAL, whereas in contrast they are not found in BAL after similar parenteral immunization, although they may be present in lung tissue. ${ }^{36,37,40,41}$ Analysis of the response to BCG, recombinant Ad85A or recombinant $M t b$ antigens, suggests that both CD8 T cells, predominantly induced by Ad85A, or CD4 T cells induced by BCG or recombinant $M t b$ antigens, can mediate early inhibition of $M t b$ growth after challenge. ${ }^{22}$ The efficacy of lungresident CD8 $\mathrm{T}$ cells in protection has been highlighted by antibody depletion and cell transfer experiments. ${ }^{41,42}$ These data indicate that LRT immunization induces an activated, antigendependent, dividing population of lung $\mathrm{T}$ cells located at the site of entry of inhaled mycobacteria, which is able to inhibit the growth of mycobacteria early after $M t b$ infection.

As well as inducing BAL antigen-specific cells, LRT immunization can generate inducible bronchus-associated lymphoid tissue. ${ }^{43,44}$ This has an important role in the immune response to many respiratory infections and recent experiments with the mucosal adjuvant Escherichia coli holotoxin LT-IIb and ESAT6 peptide antigen, show that induction of inducible bronchus-associated lymphoid tissue also contributes to protection against $M t b{ }^{45}$

Protection against $M t b$ challenge is maintained for $>6$ months after LRT immunization with Ad85A and is associated with the continued presence of antigen-specific cells in the lungs, including the BAL. Furthermore, this population retains a highly activated phenotype and the cells continue to proliferate. ${ }^{39,42}$ Proliferation is antigen dependent, indicating that antigen is retained within the lung, and the population is largely self-maintaining with little contribution from cells entering the lungs from the periphery. ${ }^{39,42}$ However, additional antigen-specific cells can be recruited if antigen or inflammatory stimuli are introduced into the lungs. ${ }^{41,46}$

\section{Lung immunity in bovines, non-human primates and humans}

In contacts of active TB patients and those with paucibacillary pulmonary disease, $M t b$ antigen-specific $\mathrm{T}$-cell responses are detected in the BAL but not in the peripheral blood, suggesting that local lung immune responses may have a vital role in protective immunity early after $M t b$ exposure. ${ }^{47}$ Interestingly, in active TB patients with higher bacterial loads, antigenspecific CD3 + T-cell responses are detected in both the BAL and blood, although responses are generally higher in the BAL near the site of infection, than the peripheral blood. ${ }^{48,49}$ These data are correlative but the potential of mucosal memory $\mathrm{T}$ cells, induced through infection or vaccination, to respond to antigen re-exposure has been elegantly demonstrated by intrapulmonary challenge with PPD in healthy adults. Rapid expansion of antigen-specific T cells was detected in the BAL
$48 \mathrm{~h}$ post-challenge only in individuals with a positive skin test to PPD. Thus, prior priming generated a memory pool capable of rapid response on subsequent antigenic re-exposure. ${ }^{30}$ However, there is little data on immune responses in the lungs following vaccination.

Non-human primate studies have demonstrated the efficacy of immunization with parenteral BCG against pulmonary $M t b$ challenge, ${ }^{50-52}$ which may be dependent on antigen-specific CD8 + T cells. ${ }^{51}$ Pulmonary administration of BCG induces higher frequencies of mucosal $\alpha \beta$ and $\gamma \delta$ T cells compared with systemic administration, which induces stronger peripheral responses $^{53}$ and remarkably, studies in 1973 in non-human primates comparing different routes of administration demonstrated improved BCG-induced protection against aerosol $\mathrm{Mtb}$ challenge in animals receiving aerosolized compared with parenteral BCG. ${ }^{54}$ However, limited data suggest that the outcome of immunization by the respiratory route may be influenced by the host species and vaccine vector, as well as the exact methodology used, for example, aerosol vs. endobronchial immunization. Non-human primates immunized by aerosol with an adenoviral vector maintained a high frequency of antigen-specific T cells in BAL for many months, whereas the response of peripheral blood mononuclear cell was transient. ${ }^{32}$ However, in macaques immunized with MVA85A there was a much less clear cut difference between lung or peripheral immune responses following aerosol or intradermal immunization. ${ }^{55}$ In bovines, boosting BCG primed animals intradermally or endobronchially with recombinant Ad85A resulted in similar peripheral blood mononuclear cell responses to PPD-B or antigen $85 \mathrm{~A}$, whereas specific immune responses were not readily detected in BAL because of a high background response in immunized animals. ${ }^{56}$

Taken together, these reports suggest that locally initiated immune responses have an important role in the early control of $M t b$ infection after exposure, although definitive evidence for their role in mediating protection in humans has yet to be obtained. Nevertheless, it seems logical that a successful vaccination strategy should aim to induce protective pulmonary cellular responses that can rapidly respond to $M t b$, as well as systemic immunity, such as that generated by parenteral BCG, which has a later effect on $M t b$ growth (Figure 1). For optimal design of novel TB vaccine strategies, the immunological conditions and innate immune signaling pathways necessary for the generation and maintenance of both these types of cellular memory responses will need to be better understood.

\section{LOCAL AND SYSTEMIC INNATE IMMUNITY}

There is increasing evidence that appropriate innate immunity is essential for protection against TB in animal models..$^{17,57-59}$ As well as inducing an antigen-specific response, parenteral BCG induces changes in gene expression in the lungs of mice more related to the innate immune system and tissue repair mechanisms than to adaptive immunity. ${ }^{60}$ Furthermore, BCG induces a state of "trained immunity" in macrophages, persisting for several months and conferring increased nonspecific protection against other infections. ${ }^{58,61}$ This state of 
heightened nonspecific immunity is dependent on nucleotidebinding oligomerization domain-containing protein 2 signaling and epigenetic mechanisms, as may be the case for other longlasting effects of microorganisms on the immune system. ${ }^{62}$

In addition to general changes in innate immunity mediated by exposure to microbes, the many differences between pulmonary and extra-pulmonary innate mechanisms need to be considered. Alveolar macrophages express higher levels of Toll-like receptor- 9 and lower surface Toll-like receptor- 2 than autologous monocytes, suggestive of a tissue-specific immune recognition and signaling system that shapes the subsequent adaptive and inflammatory immune response in the lung. ${ }^{63,64}$ Lung-specific receptors for $M t b$ such as surfactant protein A have an important role in mediating local lung immune responses by downregulating Toll-like receptor -4 and initiating a suppressive immunoregulatory environment in response to infection. ${ }^{65}$ These distinct aspects of the innate immune system are also evident in the cellular subsets of the lung. BAL of healthy individuals are predominantly composed of alveolar macrophages (90\%) and a minority population of lymphocytes (5-10\%) in comparison with peripheral blood in which neutrophils and lymphocytes predominate, with a minority monocyte population. ${ }^{66}$

Recently, the importance of activating the correct innate pathways in order to induce protective local immunity in the lungs with a subunit vaccine, has been elegantly demonstrated. Both Ad85A and VSV85A delivered to the LRT induce powerful, largely $\mathrm{CD} 8$, local immune responses but while Ad85A is protective against $M t b$, VSV85A is not. The two vaccines induce differing levels of interleukin-12 and type I interferon and this balance is critical for induction of protective or non-protective immunity. ${ }^{67}$ These data are in agreement with earlier reports that different adjuvants applied to the lungs have markedly different effects on protection against $M t b .{ }^{22,45,57}$ Although alterations in innate immune function clearly have a major effect on protection, different vectors or formulations of the same antigen can also alter the specificity of the adaptive response, targeting it to more or less protective epitopes. ${ }^{68-70}$ Thus, an effective pulmonary vaccine will depend both on the use of protective antigens and the delivery of appropriate signals by vaccine vectors or adjuvants to the innate immune system. Further experiments in primates and on human lung immunity are a priority in order to define optimal methods for harnessing human pulmonary responses against TB.

\section{HARNESSING LOCAL AND SYSTEMIC IMMUNITY}

For immunization against TB, the heterologous prime boost paradigm has received much attention because BCG provides useful protective efficacy in infants, and it is therefore an attractive strategy to try to boost this with subunit vaccines. ${ }^{71}$ In addition, parenteral prime boost regimes have been shown in animal models to induce powerful $\mathrm{T}$-cell immune responses to many different antigens. ${ }^{72-75}$ However, very few TB parenteral prime boost regimes induce significantly better protection than BCG alone $e^{12,13,71,76,77}$ and those that do often involve repeated immunization, ${ }^{12,71,78}$ a protocol that may be successful because it induces entry of immune cells into non-lymphoid tissues. ${ }^{11}$

For TB, it has been suggested that parenteral priming and mucosal boosting might have the advantage that local lung immunity would control pathogen growth in the lungs, whereas systemic immunity might control growth of organisms that escape to other tissues. In support of this, several studies have shown improved protection over BCG using parenteral BCG priming followed by respiratory mucosal boosting. ${ }^{37,67,71,79}$ It is further assumed that the efficacy of parenteral/mucosal prime boost regimes is due to the expansion of primed antigenspecific cells following boosting. However, simultaneous immunization of mice with the same vaccine by the parenteral and mucosal routes can be more protective than immunization by either route alone. Furthermore, parenteral and mucosal administration of two subunit vaccines containing different antigens, and therefore precluding a prime boost effect, can induce additive protection. ${ }^{22}$ Thus, an important outcome of combining parenteral and mucosal immunization may be to induce lung $\mathrm{T}_{\mathrm{RM}}$ resident in the airway compartment, as well as $\mathrm{T}_{\mathrm{RM}}$ present in other lung tissue compartments and memory cells in lymphoid tissues. These may have three effects on TB. First, antigen-specific $\mathrm{T}_{\mathrm{RM}}$ cells in BAL together with innate immune cells inhibit early growth of $M t b$, second $\mathrm{T}_{\mathrm{RM}}$ cells in other lung tissue compartments may inhibit further $M t b$ growth after a lag of 10-14 days. Finally, T cells from lymphoid tissue may be recruited to maintain containment in the lung or prevent dissemination or the growth of organisms after dissemination.

\section{CONCLUSIONS}

Surprisingly, in spite of the widely held view that animal models for TB vaccine testing have little predictive value, in aggregate multiple models accurately predicted the outcome of the recent MVA85A trial, ${ }^{2}$ as parenteral boosting with MVA85A alone after parenteral BCG priming, only showed a nonsignificant trend toward increased protection over BCG in two out of five animal experiments. ${ }^{5-9}$ The predictive accuracy of the models in the case of MVA85A therefore encourages the view that effective vaccine strategies in animals may also be effective in humans.

Abundant evidence suggests that immunization via the respiratory tract with the reference standard TB vaccine, BCG, can be highly effective in protecting against pulmonary challenge in mice, ${ }^{22}$ guinea pigs, ${ }^{80}$ cattle $^{81}$ and primates ${ }^{54}$ and it has been safely administered to humans by aerosol, ${ }^{82}$ as have vaccines against influenza or measles viruses. ${ }^{83}$ In mice, subunit vaccines have been shown to be protective after respiratory immunization ${ }^{36-38}$ whereas in primates, subunit viral vectored vaccines administered by aerosol are immunogenic. ${ }^{32,55}$ There is therefore good evidence on which to base human clinical trials of $\mathrm{TB}$ vaccines administered by the respiratory route. Although in animal experiments it is important to immunize the LRT, in humans it remains to be determined whether this is equally so or URT immunization will suffice, but other more important issues remain to be 
resolved. No protection data for any TB vaccine administered by the respiratory route except $B C G^{54}$ has yet been reported in primates. But the mouse data indicate the crucial importance of delivering the correct signals to the lung innate system, ${ }^{67}$ suggesting that success in primates and humans will be equally dependent on correct innate signaling as well as the specificity of the response.

Animal data indicate not only that respiratory immunization is highly effective but that it can be even more so when combined in either prime boost or simultaneous immunization regimes with parenteral immunization. ${ }^{22,67,84}$ We argue above that this may be because both early and later acting protective mechanisms are recruited by these immunization regimes.

These considerations allow the following conclusions. In aggregate, multiple animal models may be more predictive of success or failure in humans than previously believed. Animal evidence strongly supports the value of clinical trials of vaccines, and in particular BCG, administered by the respiratory route. A much better understanding of respiratory innate and adaptive immunity in humans is urgently needed and may be gained from well-planned trials of respiratory vaccines associated with experimental studies of URT and LRT immunity. Combining parenteral and respiratory immunization is promising but more investigation is needed to optimize prime/boost or simultaneous immunization strategies for humans.

\section{ACKNOWLEDGMENTS}

PCLB and EZT are supported by UK Medical Research Council grant no. 60071235. SS and AL are supported by the Wellcome Trust and the UK National Institute for Health Research.

\section{DISCLOSURE}

The authors declared no conflict of interest.

c) 2014 Society for Mucosal Immunology

\section{REFERENCES}

1. Bishai, W., Sullivan, Z., Bloom, B.R. \& Andersen, P. Bettering BCG: a tough task for a TB vaccine?. Nat. Med 19, 410-411 (2013).

2. Tameris, M.D. et al. Safety and efficacy of MVA85A, a new tuberculosis vaccine, in infants previously vaccinated with BCG: a randomised, placebo-controlled phase $2 b$ trial. Lancet 381, 1021-1028 (2013).

3. Tameris, M. et al. Lessons learnt from the first efficacy trial of a new infant tuberculosis vaccine since BCG. Tuberculosis (Edinb) 93 , 143-149 (2013).

4. Lalvani, A., Sridhar, S. \& Fordham von Reyn, C. Tuberculosis vaccines: time to reset the paradigm? Thorax, doi:10.1136/thoraxjnl-2013-203456 2013 (e-pub ahead of print).

5. Tchilian, E.Z. et al. Immunogenicity and protective efficacy of prime-boost regimens with recombinant (delta)ureC hly + Mycobacterium bovis BCG and modified vaccinia virus ankara expressing $M$. tuberculosis antigen $85 \mathrm{~A}$ against murine tuberculosis. Infect. Immun 77, 622-631 (2009).

6. Williams, A. et al. Evaluation of vaccines in the EU TB Vaccine Cluster using a guinea pig aerosol infection model of tuberculosis. Tuberculosis (Edinb) 85, 29-38 (2005).

7. Vordermeier, H.M. et al. Viral booster vaccines improve Mycobacterium bovis BCG-induced protection against bovine tuberculosis. Infect. Immun 77, 3364-3373 (2009).

8. Verreck, F.A. et al. MVA.85A boosting of BCG and an attenuated, phoP deficient $M$. tuberculosis vaccine both show protective efficacy against tuberculosis in rhesus macaques. PLoS One 4, e5264 (2009).
9. Sharpe, S.A. et al. Establishment of an aerosol challenge model of tuberculosis in rhesus macaques and an evaluation of endpoints for vaccine testing. Clin. Vaccine Immunol 17, 1170-1182 (2010).

10. McShane, H. et al. Recombinant modified vaccinia virus Ankara expressing antigen 85 A boosts BCG-primed and naturally acquired antimycobacterial immunity in humans. Nat. Med 10, 1240-1244 (2004).

11. Tatsis, N., Lin, S.W., Harris-McCoy, K., Garber, D.A., Feinberg, M.B. \& Ertl, H.C. Multiple immunizations with adenovirus and MVA vectors improve CD8 + T cell functionality and mucosal homing. Virology 367 , 156-167 (2007).

12. Lin, P.L. et al. The multistage vaccine $\mathrm{H} 56$ boosts the effects of $B C G$ to protect cynomolgus macaques against active tuberculosis and reactivation of latent Mycobacterium tuberculosis infection. J. Clin. Invest 122, 303-314 (2012).

13. Lindenstrom, T. et al. Tuberculosis subunit vaccination provides long-term protective immunity characterized by multifunctional CD4 memory T cells. J. Immunol 182, 8047-8055 (2009).

14. Lindenstrom, T., Knudsen, N.P., Agger, E.M. \& Andersen, P. Control of chronic Mycobacterium tuberculosis infection by CD4 KLRG1- IL-2secreting central memory cells. J. Immunol 190, 6311-6319 (2013).

15. Khader, S.A. et al. IL-23 and IL-17 in the establishment of protective pulmonary $\mathrm{CD} 4+\mathrm{T}$ cell responses after vaccination and during Mycobacterium tuberculosis challenge. Nat. Immunol 8, 369-377 (2007).

16. Vordermeier, H.M. et al. Cellular immune responses induced in cattle by heterologous prime-boost vaccination using recombinant viruses and bacille Calmette-Guerin. Immunology 112, 461-470 (2004).

17. Ernst, J.D. The immunological life cycle of tuberculosis. Nat. Rev. Immunol. 12, 581-591 (2012).

18. Shaler, C.R., Horvath, C., Lai, R. \& Xing, Z. Understanding delayed T-cell priming, lung recruitment, and airway luminal T-cell responses in host defense against pulmonary tuberculosis. Clin. Dev. Immunol 2012, 628293 (2012).

19. Reiley, W.W. et al. ESAT-6-specific CD4 T cell responses to aerosol Mycobacterium tuberculosis infection are initiated in the mediastinal lymph nodes. Proc. Natl. Acad. Sci. USA 105, 10961-10966 (2008).

20. Cooper, A.M. Cell-mediated immune responses in tuberculosis. Annu. Rev. Immunol. 27, 393-422 (2009).

21. Horvath, C.N., Shaler, C.R., Jeyanathan, M., Zganiacz, A. \& Xing, Z. Mechanisms of delayed anti-tuberculosis protection in the lung of parenteral BCG-vaccinated hosts: a critical role of airway luminal T cells. Mucosal. Immunol 5, 420-431 (2012).

22. Tchilian, E.Z. et al. Simultaneous immunization against tuberculosis. PLoS One 6, e27477 (2011)

23. Winslow, G.M., Cooper, A., Reiley, W., Chatterjee, M. \& Woodland, D.L. Early T-cell responses in tuberculosis immunity. Immunol. Rev. 225, 284299 (2008).

24. Connor, L.M. et al. A key role for lung-resident memory lymphocytes in protective immune responses after BCG vaccination. Eur. J. Immuno/ 40, 2482-2492 (2010).

25. Jiang, X., Clark, R.A., Liu, L., Wagers, A.J., Fuhlbrigge, R.C. \& Kupper, T.S. Skin infection generates non-migratory memory CD8 $+\mathrm{T}(\mathrm{RM})$ cells providing global skin immunity. Nature 483, 227-231 (2012).

26. Wakim, L.M., Gupta, N., Mintern, J.D. \& Villadangos, J.A. Enhanced survival of lung tissue-resident memory CD $8(+)$ Tcells during infection with influenza virus due to selective expression of IFITM3. Nat. Immunol 14, 238-245 (2013).

27. Liang, S., Mozdzanowska, K., Palladino, G. \& Gerhard, W. Heterosubtypic immunity to influenza type A virus in mice. Effector mechanisms and their longevity. J. Immunol 152, 1653-1661 (1994).

28. Hogan, R.J. et al. Activated antigen-specific CD8 + T cells persist in the lungs following recovery from respiratory virus infections. J. Immunol 166, 1813-1822 (2001)

29. Purwar, R., Campbell, J., Murphy, G., Richards, W.G., Clark, R.A. \& Kupper, T.S. Resident memory T cells (T(RM)) are abundant in human lung: diversity, function, and antigen specificity. PLoS One 6, e16245 (2011).

30. Walrath, J., Zukowski, L., Krywiak, A. \& Silver, R.F. Resident Th1-like effector memory cells in pulmonary recall responses to Mycobacterium tuberculosis. Am. J. Respir. Cell. Mol. Biol 33, 48-55 (2005).

31. Ronan, E.O., Lee, L.N., Tchilian, E.Z. \& Beverley, P.C. Nasal associated lymphoid tissue (NALT) contributes little to protection against aerosol 
challenge with Mycobacterium tuberculosis after immunisation with a recombinant adenoviral vaccine. Vaccine 28, 5179-5184 (2010).

32. Song, K. et al. Genetic immunization in the lung induces potent local and systemic immune responses. Proc. Natl. Acad. Sci. USA 107, 22213-22218 (2010).

33. Bourdin, A., Gras, D., Vachier, I. \& Chanez, P. Upper airway $x$ 1: allergic rhinitis and asthma: united disease through epithelial cells. Thorax 64, 999-1004 (2009).

34. Hurst, J.R. Upper airway. 3: sinonasal involvement in chronic obstructive pulmonary disease. Thorax 65, 85-90 (2010).

35. Wang, J. et al. Single mucosal, but not parenteral, immunization with recombinant adenoviral-based vaccine provides potent protection from pulmonary tuberculosis. J. Immunol 173, 6357-6365 (2004).

36. Santosuosso, M., Zhang, X., McCormick, S., Wang, J., Hitt, M. \& Xing, Z. Mechanisms of mucosal and parenteral tuberculosis vaccinations: adenoviral-based mucosal immunization preferentially elicits sustained accumulation of immune protective CD4 and CD8 T cells within the airway lumen. J. Immunol 174, 7986-7994 (2005).

37. Forbes, E.K. et al. Multifunctional, high-level cytokine-producing Th1 cells in the lung, but not spleen, correlate with protection against Mycobacterium tuberculosis aerosol challenge in mice. J. Immunol 181, 4955-4964 (2008)

38. Goonetilleke, N.P., McShane, H., Hannan, C.M., Anderson, R.J., Brookes, R.H. \& Hill, A.V. Enhanced immunogenicity and protective efficacy against Mycobacterium tuberculosis of bacille Calmette-Guerin vaccine using mucosal administration and boosting with a recombinant modified vaccinia virus Ankara. J. Immunol 171, 1602-1609 (2003).

39. Ronan, E.O., Lee, L.N., Beverley, P.C. \& Tchilian, E.Z. Immunization of mice with a recombinant adenovirus vaccine inhibits the early growth of Mycobacterium tuberculosis after infection. PLoS One 4, e8235 (2009).

40. Santosuosso, M., McCormick, S., Zhang, X., Zganiacz, A. \& Xing, Z. Intranasal boosting with an adenovirus-vectored vaccine markedly enhances protection by parenteral Mycobacterium bovis BCG immunization against pulmonary tuberculosis. Infect. Immun 74, 4634-4643 (2006).

41. Lee, L.N. et al. CXCR6 is a marker for protective antigen-specific cells in the lungs after intranasal immunization against Mycobacterium tuberculosis. Infect. Immun 79, 3328-3337 (2011).

42. Jeyanathan, M. et al. Murine airway luminal anti-tuberculosis memory CD8 T cells by mucosal immunization are maintained via antigen-driven in situ proliferation, independent of peripheral T cell recruitment. Am. J. Respir. Crit. Care Med 181, 862-872 (2010).

43. Xu, B. et al. Lymphocyte homing to bronchus-associated lymphoid tissue (BALT) is mediated by L-selectin/PNAd, alpha4beta1 integrin/NCAM-1, and LFA-1 adhesion pathways. J. Exp. Med 197, 1255-1267 (2003).

44. Moyron-Quiroz, J.E. et al. Role of inducible bronchus associated lymphoid tissue (BALT) in respiratory immunity. Nat. Med 10, 927-934 (2004).

45. Gopal, R. et al. Interleukin-17-dependent CXCL13 mediates mucosal vaccine-induced immunity against tuberculosis. Mucosal Immunol 6, 972-984 (2013).

46. Chen, X. et al. Regulation of TB vaccine-induced airway luminal T cells by respiratory exposure to endotoxin. PLoS One 7, e41666 (2012).

47. Schwander, S.K. et al. Pulmonary mononuclear cell responses to antigens of Mycobacterium tuberculosis in healthy household contacts of patients with active tuberculosis and healthy controls from the community. J. Immunol 165, 1479-1485 (2000).

48. Schwander, S.K. et al. T lymphocytic and immature macrophage alveolitis in active pulmonary tuberculosis. J. Infect. Dis 173, 1267-1272 (1996).

49. Boras, Z., Juretic, A., Gagro, A. \& Pavelic, L. Cytokine profile of $t$ lymphocytes from peripheral blood and bronchoalveolar lavage fluid in patients with active pulmonary tuberculosis. Scand. J. Immunol 65, 257-264 (2007).

50. Larsen, M.H. et al. Efficacy and safety of live attenuated persistent and rapidly cleared Mycobacterium tuberculosis vaccine candidates in non-human primates. Vaccine 27, 4709-4717 (2009).

51. Chen, C.Y. et al. A critical role for CD8 T cells in a nonhuman primate model of tuberculosis. PLoS Pathog 5, e1000392 (2009).

52. Langermans, J.A. et al. Protection of macaques against Mycobacterium tuberculosis infection by a subunit vaccine based on a fusion protein of antigen 85B and ESAT-6. Vaccine 23, 2740-2750 (2005).
53. Lai, X. et al. Immune biology of macaque lymphocyte populations during mycobacterial infection. Clin. Exp. Immunol 133, 182-192 (2003).

54. Barclay, W.R. et al. Protection of monkeys against airborne tuberculosis by aerosol vaccination with bacillus Calmette-Guerin. Am. Rev. Respir. Dis. 107, 351-358 (1973).

55. White, A.D. et al. An evaluation of the safety and immunogenicity of a candidate TB vaccine, MVA85A, delivered by aerosol to the lungs of macaques. Clin. Vaccine. Immunol 20, 663-672 (2013).

56. Whelan, A. et al. Immunogenicity comparison of the intradermal or endobronchial boosting of BCG vaccinates with Ad5-85 A. Vaccine 30, 6294-6300 (2012).

57. Antonelli, L.R. et al. Intranasal Poly-IC treatment exacerbates tuberculosis in mice through the pulmonary recruitment of a pathogen-permissive monocyte/macrophage population. J. Clin. Invest 120, 1674-1682 (2010).

58. Cooper, A.M., Mayer-Barber, K.D. \& Sher, A. Role of innate cytokines in mycobacterial infection. Mucosal Immunol 4, 252-260 (2011).

59. Potian, J.A., Rafi, W., Bhatt, K., McBride, A., Gause, W.C. \& Salgame, P. Preexisting helminth infection induces inhibition of innate pulmonary antituberculosis defense by engaging the IL-4 receptor pathway. J Exp Med 208, 1863-1874 (2011).

60. Aranday Cortes, E., Kaveh, D., Nunez-Garcia, J., Hogarth, P.J. \& Vordermeier, H.M. Mycobacterium bovis-BCG vaccination induces specific pulmonary transcriptome biosignatures in mice. PLoS One 5, e11319 (2010)

61. Kleinnijenhuis, J. et al. Bacille Calmette-Guerin induces NOD2dependent nonspecific protection from reinfection via epigenetic reprogramming of monocytes. Proc. Natl. Acad. Sci. USA 109, 17537-17542 (2012).

62. Ivanov, I.I. \& Littman, D.R. Modulation of immune homeostasis by commensal bacteria. Curr. Opin. Microbiol. 14, 106-114 (2011).

63. Juarez, E., Nunez, C., Sada, E., Ellner, J.J., Schwander, S.K. \& Torres, M. Differential expression of Toll-like receptors on human alveolar macrophages and autologous peripheral monocytes. Respir. Res 11, 2 (2010).

64. Nguyen, H.A., Rajaram, M.V., Meyer, D.A. \& Schlesinger, L.S. Pulmonary surfactant protein $\mathrm{A}$ and surfactant lipids upregulate IRAK-M, a negative regulator of TLR-mediated inflammation in human macrophages. Am. J. Physiol. Lung Cell. Mol. Physiol 303, L608-L616 (2012).

65. Henning, L.N., Azad, A.K., Parsa, K.V., Crowther, J.E., Tridandapani, S. \& Schlesinger, L.S. Pulmonary surfactant protein A regulates TLR expression and activity in human macrophages. J. Immunol. 180, 7847-7858 (2008).

66. Schwander, S. \& Dheda, K. Human lung immunity against Mycobacterium tuberculosis: insights into pathogenesis and protection. Am. J. Respir. Crit. Care Med 183, 696-707 (2011).

67. Jeyanathan, M. et al. Differentially imprinted innate immunity by mucosal boost vaccination determines antituberculosis immune protective outcomes, independent of T-cell immunity. Mucosal Immunol 6, 612-625 (2013).

68. Bennekov, T., Dietrich, J., Rosenkrands, I., Stryhn, A., Doherty, T.M. \& Andersen, P. Alteration of epitope recognition pattern in Ag85B and ESAT-6 has a profound influence on vaccine-induced protection against Mycobacterium tuberculosis. Eur. J. Immunol 36, 3346-3355 (2006).

69. Hansen, S.G. et al. Cytomegalovirus vectors violate CD8 + T cell epitope recognition paradigms. Science 340, 1237874 (2013).

70. Tchilian, E., Ahuja, D., Hey, A., Jiang, S. \& Beverley, P. Immunization with different formulations of Mycobacterium tuberculosis antigen $85 \mathrm{~A}$ induces immune responses with different specificity and protective efficacy. Vaccine 31, 4624-4631 (2013).

71. Brennan, M.J. et al. Preclinical evidence for implementing a prime-boost vaccine strategy for tuberculosis. Vaccine 30, 2811-2823 (2012).

72. Woodland, D.L. Jump-starting the immune system: prime-boosting comes of age. Trends Immunol 25, 98-104 (2004).

73. Draper, S.J. \& Heeney, J.L. Viruses as vaccine vectors for infectious diseases and cancer. Nat. Rev. Microbiol 8, 62-73 (2010).

74. Robinson, H.L. \& Amara, R.R. T cell vaccines for microbial infections. Nat. Med 11, S25-S32 (2005).

75. Hansen, S.G. et al. Profound early control of highly pathogenic SIV by an effector memory T-cell vaccine. Nature 473, 523-527 (2011).

76. Aagaard, C. et al. A multistage tuberculosis vaccine that confers efficient protection before and after exposure. Nat Med 17, 189-194 (2011). 
77. Reed, S.G. et al. Defined tuberculosis vaccine, Mtb72F/AS02A, evidence of protection in cynomolgus monkeys. Proc Natl Acad Sci USA 106, 2301-2306 (2009).

78. Billeskov, R., Elvang, T.T., Andersen, P.L. \& Dietrich, J. The HyVac4 subunit vaccine efficiently boosts BCG-primed anti-mycobacterial protective immunity. PLoS One 7, e39909 (2012).

79. Xing, Z., McFarland, C.T., Sallenave, J.M., Izzo, A., Wang, J. \& McMurray, D.N. Intranasal mucosal boosting with an adenovirus-vectored vaccine markedly enhances the protection of BCG-primed guinea pigs against pulmonary tuberculosis. PLoS One 4, e5856 (2009).

80. Cohn, M.L., Davis, C.L. \& Middlebrook, G. Airborne immunization against tuberculosis. Science 128, 1282-1283 (1958).

81. Buddle, B.M. et al. Protection of cattle from bovine tuberculosis by vaccination with BCG by the respiratory or subcutaneous route, but not by vaccination with killed Mycobacterium vaccae. Res. Vet. Sci 59, 10-16 (1995).
82. Rosenthal, S.R., McEnery, J.T. \& Raisys, N. Aerogenic BCG vaccination against tuberculosis in animal and human subjects. J. Asthma Res 5, 309-323 (1968).

83. Cutts, F.T., Clements, C.J. \& Bennett, J.V. Alternative routes of measles immunization: a review. Biologicals 25, 323-338 (1997).

84. Xing, Z. \& Charters, T.J. Heterologous boost vaccines for bacillus Calmette-Guerin prime immunization against tuberculosis. Expert Rev. Vaccines 6, 539-546 (2007).

(1) (9) This work is licensed under the Creative Commons Attribution-NonCommercial-No Derivative Works 3.0 Unported License. To view a copy of this license, visit http://creativecommons.org/licenses/by-nc-nd/3.0/ 